\title{
Mechanical Vibration Signal Denoising Using Quantum-Inspired Standard Deviation Based on Subband Based Gaussian Mixture Model
}

\author{
Aidong Xu, Wenqi Huang (D), Peng Li, Huajun Chen, Jiaxiao Meng, and Xiaobin Guo
}

Electric Power Research Institute, China Southern Power Grid, Guangzhou 510663, China

Correspondence should be addressed to Wenqi Huang; gzhwq88@163.com

Received 26 July 2017; Revised 18 February 2018; Accepted 21 February 2018; Published 27 March 2018

Academic Editor: Matteo Filippi

Copyright (C) 2018 Aidong Xu et al. This is an open access article distributed under the Creative Commons Attribution License, which permits unrestricted use, distribution, and reproduction in any medium, provided the original work is properly cited.

\begin{abstract}
Aiming at improving noise reduction effect for mechanical vibration signal, a Gaussian mixture model (SGMM) and a quantuminspired standard deviation (QSD) are proposed and applied to the denoising method using the thresholding function in wavelet domain. Firstly, the SGMM is presented and utilized as a local distribution to approximate the wavelet coefficients distribution in each subband. Then, within Bayesian framework, the maximum a posteriori (MAP) estimator is employed to derive a thresholding function with conventional standard deviation (CSD) which is calculated by the expectation-maximization (EM) algorithm. However, the CSD has a disadvantage of ignoring the interscale dependency between wavelet coefficients. Considering this limit for the CSD, the quantum theory is adopted to analyze the interscale dependency between coefficients in adjacent subbands, and the QSD for noise-free wavelet coefficients is presented based on quantum mechanics. Next, the QSD is constituted for the CSD in the thresholding function to shrink noisy coefficients. Finally, an application in the mechanical vibration signal processing is used to illustrate the denoising technique. The experimental study shows the SGMM can model the distribution of wavelet coefficients accurately and QSD can depict interscale dependency of wavelet coefficients of true signal quite successfully. Therefore, the denoising method utilizing the SGMM and QSD performs better than others.
\end{abstract}

\section{Introduction}

With the science and technology developing, the mechanical equipment is becoming more and more complicated, which indicates that intelligent controlling and monitoring methods are necessary for machineries. The signal is widely used in mechanical system for controlling and monitoring [1-5] and helps to optimize the mechanical equipment management. The vibration signal among the most popular signals is applied to the mechanical information extraction. However, noise inevitably exists in mechanical vibration signal. Thus the study of noise reduction has attracted sustained attention in the past few decades, and estimating the noisefree signal coefficients in the wavelet domain is a practical way while it is difficult to recover noise-free signal in the time domain. Improved from VisuShrink, SureShrink, and BayesShrink, a fair amount of wavelets based on denoising techniques using thresholding functions has been developed for one-dimensional [6-8], two-dimensional [9-12], and three-dimensional $[13,14]$ signal. It has been demonstrated that the thresholding function based denoising algorithms utilizing local probability density function (PDF) [15] or utilizing interscale dependency of wavelet coefficients $[16,17]$ are among the best.

The noise reduction algorithm using the thresholding function can be considered as estimating the noise-free wavelet coefficients as accurately as possible in a Bayesian framework [15]. The maximum a posteriori (MAP) Bayesian estimator is often employed to derive the thresholding function based on an appropriate prior knowledge of noisefree wavelet coefficients. So, the adopted distribution for the wavelet coefficients plays a key role in the performance of mechanical vibration signal denoising. Due to the versatility of the Gaussian mixture model (GMM) in characterizing statistical behavior in the wavelet domain, the GMM has been extensively applied in modeling the PDF of wavelet 
coefficients accurately [18], and the thresholding function derived from GMM performances shows better shrinking coefficients than those based on Gaussian model and Laplacian model. However, the GMM is mostly used for twodimensional image rather than one-dimensional mechanical signal processing; it is necessary to do some research on onedimensional signal processing for wider application of GMM. After wavelet decomposition, different subbands will present different wavelet coefficients. It is logical to take the level of subband into account while the GMM is employed to model the coefficient distribution in each level. In order to enhance the denoising effect, a subband based on Gaussian mixture model (SGMM) is proposed as a local PDF for each wavelet coefficient in its neighborhood.

The standard deviation of the clean wavelet coefficients is an important parameter in SGMM. The conventional standard deviation (CSD) is mostly used to estimate the standard deviation of noise-free coefficients and it will finally appear in the thresholding function after MAP estimation based on SGMM, which will strongly influence the noise reduction effect. The CSD of clean wavelet coefficients is computed via an statistical way in its neighborhood [17], which ignores the interscale dependency between adjacent subbands. It has been revealed that denoising algorithms $[16,17,19]$ which consider the influence of interscale dependency of wavelet coefficients performs better in denoising, whereas the studies shown in $[16,17,19]$ consider interscale dependency in statistic distribution model and use the CSD of clean wavelet coefficients to handle wavelet. Since many works have shown strong evidences that wavelet coefficients produced by true signal exhibit significant dependencies on subsequent locations, in this paper the interscale dependency is taken into account from another perspective, which indicates that we will integrate the interscale dependency into the standard deviation. In order to evaluate the interscale dependency quantitatively, the quantum theory is employed due to its special nature.

Quantum theory is an ongoing research in the fields of quick search [20], optimization [21, 22], clustering [23], and key distribution [24]. The work of incorporating quantum mechanics into other theories has stimulated the researches of quantum-inspired algorithms and their applications. Relying on the basic principles of quantum mechanics, a qubit described as a superposition can represent all states simultaneously that a datum may generate [21, 22]. A wavelet coefficient consists of the noise-free coefficient and the noise coefficient; in other words, it is the superposition of signal and noise. Therefore, quantum theory is potential to depict the interscale dependency between subbands in the same location. We will try to derive a quantum-inspired standard deviation (QSD) for noise-free wavelet coefficients in order to improve noise reduction effect of denoising method with CSD.

From the above, in this paper the clean coefficients are estimated from the noisy data observations considering both local PDF using SGMM and interscale dependency using QSD while processing the wavelet coefficients with Bayesian estimation techniques. Using the SGMM with QSD has two advantages: (1) One better prior distribution of wavelet coefficients will result in one better thresholding function. The SGMM can model the distribution of wavelet coefficients more accurately in each subband; (2) the interscale dependency is a basic property of wavelet coefficients and the QSD considers the interscale dependency quantitatively. Combining the SGMM with QSD thus will elicit an exact thresholding function which will reduce noise professionally.

The remainder of the paper is organized as follows. In Section 2 the SGMM is presented and the thresholding function based on local SGMM with CSD using MAP estimator is derived. In Section 3, the quantum theory is employed to evaluate the interscale dependency between adjacent subbands quantitatively. The QSD integrating quantum mechanics is presented and substituted for CSD in the thresholding function. Then the procedures of the denoising algorithm using QSD are presented. In Section 4, the proposed denoising algorithm is applied and experimental results are discussed. Finally, Section 5 offers some conclusions.

\section{Bayesian Denoising Based on SGMM with CSD}

2.1. Bayesian Denoising Based on Gaussian PDF. In this paper, a mechanical vibration signal corrupted by additive white Gaussian noise (AWGN) with zero mean will be considered. For ease of clear expression for deriving thresholding function using GMM as a local distribution, the thresholding function based on Gaussian PDF with local parameters is given first in this section. After wavelet transforming, the wavelet coefficients satisfy $y=w+n$, where $y$ is the noisy wavelet coefficient of sampled mechanical signal, $w$ is the noise-free wavelet coefficient to be computed, and $n$ is noise coefficient, which is independent white zero-mean Gaussian one.

Dual-tree complex wavelet transform (DTCWT) can reduce spectral aliasing for vibration signals and enjoys nearly shift invariance compared to other wavelets, which are attractive properties favorable to signal processing $[7,25,26]$. Owing to these advantages, DTCWT is utilized to conduct study in the next couple of sections. Note that DTCWT coefficients are complex, so we assume real part and imaginary part of coefficients are independent, for the real part and imaginary part are produced by two different trees. In the following sections, in order to facilitate the formulations, the DTCWT coefficients referred to are the real part. But the obtained equations and contents are also suitable for imaginary part.

Given random variable $Y$ for $Y=y$ and letting $p_{w \mid y}(w \mid$ $y$ ) denote the conditional PDF of random variable $W$ for $W=w$, the standard MAP estimation for $w(y)$ from the noisy observation $y$ is given below:

$$
\widehat{w}(y)=\underset{w}{\arg \max } p_{w \mid y}(w \mid y) .
$$

The thresholding based denoising method employing local PDF can threshold each sampling point of each subband separately; thus its denoising ability is more powerful [15]. With that in the mind, the supposed local PDF for each wavelet coefficient is different; the equation $y(k)=w(k)+$ $n(k)$ is needed, where $k=1, \ldots, N_{s}$ and $N_{s}$ is the quantity 
of DTCWT coefficients in the sth subband. Thus the MAP estimation of $w(k)$ is expressed as

$$
\widehat{w}(k)=\underset{w(k)}{\arg \max } p_{w(k) \mid y(k)}(w(k) \mid y(k)) .
$$

Suppose the PDF of the noise-free DTCWT coefficient $w(k) p_{w(k)}(w(k))$ is Gaussian with the standard deviation $\sigma(k)$.

$$
\begin{aligned}
p_{w(k)}(w(k)) & =\operatorname{Gaussian}(w(k), \sigma(k)) \\
& =\frac{1}{\sigma \sqrt{2 \pi}} \exp \left(-\frac{w^{2}(k)}{2 \sigma^{2}(k)}\right) .
\end{aligned}
$$

Suppose the PDF of noise coefficient $n(k) p_{n(k)}(n(k))$ is Gaussian with the standard deviation $\sigma_{n}$. The following formula can be adopted:

$$
\begin{aligned}
p_{n(k)}(n(k)) & =\operatorname{Gaussian}\left(n(k), \sigma_{n}\right) \\
& =\frac{1}{\sigma \sqrt{2 \pi}} \exp \left(-\frac{n^{2}(k)}{2 \sigma_{n}^{2}}\right) .
\end{aligned}
$$

Using Bayes rule, the following thresholding function is derived:

$$
\widehat{w}(k)=\frac{\sigma^{2}(k)}{\sigma^{2}(k)+\sigma_{n}^{2}} y(k) .
$$

2.2. Thresholding Function Based on SGMM. Based on the previous part of thresholding function using Gaussian PDF, this part focuses on deriving thresholding function based on SGMM as local PDF for each noise-free coefficient. A GMM which is the sum of two Gaussian PDFs [27] is formulated as

$$
\begin{aligned}
p_{w}(w)= & a \operatorname{Gaussian}\left(w, \sigma_{1}\right) \\
& +(1-a) \operatorname{Gaussian}\left(w, \sigma_{2}\right) \\
= & a \frac{1}{\sigma_{1} \sqrt{2 \pi}} \exp \left(-\frac{w^{2}}{2 \sigma_{1}^{2}}\right) \\
& +(1-a) \frac{1}{\sigma_{2} \sqrt{2 \pi}} \exp \left(-\frac{w^{2}}{2 \sigma_{2}^{2}}\right) .
\end{aligned}
$$

As the GMM has more parameters than Gaussian PDF $\operatorname{Gaussian}\left(w, \sigma_{1}\right)$ or $\operatorname{Gaussian}\left(w, \sigma_{2}\right)$, it is more flexible for matching the histogram of a given dataset. Inspired by (6), assuming the noise-free wavelet coefficients distribution as a mixture PDF using local parameters under the subbands $H_{0}$ and $H_{1}$, the SGMM is presented. The SGMM for noise-free DTCWT coefficients is as follows:

$$
\begin{aligned}
p_{w(k)}(w(k))=a(k) p_{1}(w(k))+(1-a(k)) p_{2}(w(k)) \\
=a(k) \text { Gaussian }\left(w(k), \sigma_{1}(k)\right) \\
\quad+(1-a(k)) \text { Gaussian }\left(w(k), \sigma_{2}(k)\right)
\end{aligned}
$$

$$
\begin{aligned}
= & a(k) \frac{1}{\sigma_{1}(k) \sqrt{2 \pi}} \exp \left(-\frac{w^{2}(k)}{2 \sigma_{1}^{2}(k)}\right) \\
& +(1-a(k)) \frac{1}{\sigma_{2}(k) \sqrt{2 \pi}} \exp \left(-\frac{w^{2}(k)}{2 \sigma_{2}^{2}(k)}\right),
\end{aligned}
$$

where $w_{1}$ is the coefficient under the subband $H_{0}$ and $w_{2}$ is the coefficient under the subband $H_{1}$. Suppose $\widehat{w}_{1}(k)$ is estimation of $w(k)$ under the subband $H_{0}$ using the MAP estimator, while $\widehat{w}_{2}(k)$ is estimation of $w(k)$ under the subband $H_{1}$ using MAP estimator. Equation (7) is used as follows: firstly, $a(k)$ and $\left(1 / \sigma_{1}(k) \sqrt{2 \pi}\right) \exp \left(-w^{2}(k) / 2 \sigma_{1}^{2}(k)\right)$ are calculated based on $H_{0}$; the $\left(1 / \sigma_{2}(k) \sqrt{2 \pi}\right) \exp \left(-w^{2}(k) / 2 \sigma_{2}^{2}(k)\right)$ is calculated based on $H_{1}$. Then (7) is applied to coefficients in both $H_{0}$ and $H_{1}$. Therefore, in (7) $w(k)$ denotes all noise-free coefficients from $H_{0}$ and $H_{1}$.

It is natural to think $w(k)$ is the combination of $\widehat{w}_{1}(k)$ and $\widehat{w}_{2}(k)$. In fact, the following equation is satisfied:

$$
\widehat{w}(k)=p_{a}(y(k)) \widehat{w}_{1}(k)+p_{1-a}(y(k)) \widehat{w}_{2}(k),
$$

where $p\left(H_{0} \mid y(k)\right):=p_{a}(y(k))$ and $p\left(H_{1} \mid y(k)\right):=$ $p_{1-a}(y(k))$. Here, $y(k)$ denotes all noisy coefficients from $H_{0}$ and $H_{1}$. Since the subband is limited in $H_{0}$ and $H_{1}$, coefficients are Gaussian with standard deviations $\sigma_{1}(k)$ and $\sigma_{2}(k)$, respectively; thresholding function (5) can be employed to compute $\widehat{w}_{1}(k)$ and $\widehat{w}_{2}(k)$. In this case, (8) can be further written as

$$
\begin{aligned}
\widehat{w}(k)= & p_{a}(y(k)) \frac{\sigma_{1}^{2}(k)}{\sigma_{1}^{2}(k)+\sigma_{n}^{2}} y(k) \\
& +p_{1-a}(y(k)) \frac{\sigma_{2}^{2}(k)}{\sigma_{2}^{2}(k)+\sigma_{n}^{2}} y(k) .
\end{aligned}
$$

In order to calculate $p_{a}(y(k))$ and $p_{1-a}(y(k))$, the following rule is used:

$$
\begin{aligned}
& p_{a}(y(k))=\frac{a(k) g_{1}(y(k))}{a(k) g_{1}(y(k))+(1-a(k)) g_{2}(y(k))} \\
& p_{1-a}(y(k)) \\
& \quad=\frac{(1-a(k)) g_{2}(y(k))}{a(k) g_{1}(y(k))+(1-a(k)) g_{2}(y(k))},
\end{aligned}
$$

where $p\left(y(k) \mid H_{0}\right):=g_{1}(y(k))$ denotes the PDF of $y(k)$ according to $H_{0}$ and $p\left(y(k) \mid H_{1}\right):=g_{2}(y(k))$ denotes the PDF of $y(k)$ according to $H_{1}$. So (9) can be formulated as

$$
\begin{aligned}
\widehat{w}(k)= & \frac{a(k) g_{1}(y(k))}{a(k) g_{1}(y(k))+(1-a(k)) g_{2}(y(k))} \\
& \times \frac{\sigma_{1}^{2}(k)}{\sigma_{1}^{2}(k)+\sigma_{n}^{2}} y(k) \\
& +\frac{(1-a(k)) g_{2}(y(k))}{a(k) g_{1}(y(k))+(1-a(k)) g_{2}(y(k))} \\
& \times \frac{\sigma_{2}^{2}(k)}{\sigma_{2}^{2}(k)+\sigma_{n}^{2}} y(k) .
\end{aligned}
$$


Noisy coefficients are the sum of noise-free coefficients and Gaussian noise and they satisfy the convolution

$$
\begin{aligned}
p_{y(k)}(y(k)) \\
=\left(a(k) p_{1}(y(k))+(1-a(k)) p_{2}(y(k))\right) \\
\quad * p_{n}(y(k)) \\
=a(k) p_{1}(y(k)) * p_{n}(y(k)) \\
\quad+(1-a(k)) p_{2}(y(k)) * p_{n}(y(k)) \\
=a(k) g_{1}(y(k))+(1-a(k)) g_{2}(y(k)) .
\end{aligned}
$$

Because $w(k)$ follows the SGMM with local parameters according to (7), Gaussian PDFs $p_{1}(w(k))$ and $p_{2}(w(k))$ include parameters $\sigma_{1}$ and $\sigma_{2}$. On this basis, $g_{1}(y(k))$ and $g_{2}(y(k))$ in (12) can be expressed as

$$
\begin{aligned}
g_{1}(y(k))= & \text { Gaussian }\left(y(k), \sigma_{1}(k)\right) \\
& * \operatorname{Gaussian}\left(y(k), \sigma_{n}(k)\right) \\
= & \operatorname{Gaussian}\left(y(k), \sqrt{\sigma_{1}^{2}(k)+\sigma_{n}^{2}(k)}\right) \\
g_{2}(y(k))= & \text { Gaussian }\left(y(k), \sigma_{2}(k)\right) \\
& * \text { Gaussian }\left(y(k), \sigma_{n}(k)\right) \\
= & \text { Gaussian }\left(y(k), \sqrt{\sigma_{2}^{2}(k)+\sigma_{n}^{2}(k)}\right) .
\end{aligned}
$$

Therefore, $p_{a}(y(k))$ and $p_{1-a}(y(k))$ in (10) can be expressed as

$$
\begin{aligned}
p_{a}(y(k)) & =\frac{a(k) \text { Gaussian }\left(y(k), \sqrt{\sigma_{1}^{2}(k)+\sigma_{n}^{2}(k)}\right)}{a(k) \text { Gaussian }\left(y(k), \sqrt{\sigma_{1}^{2}(k)+\sigma_{n}^{2}(k)}\right)+(1-a(k)) \text { Gaussian }\left(y(k), \sqrt{\sigma_{2}^{2}(k)+\sigma_{n}^{2}(k)}\right)} \\
p_{1-a}(y(k)) & =\frac{(1-a(k)) \text { Gaussian }\left(y(k), \sqrt{\sigma_{2}^{2}(k)+\sigma_{n}^{2}(k)}\right)}{a(k) \text { Gaussian }\left(y(k), \sqrt{\sigma_{1}^{2}(k)+\sigma_{n}^{2}(k)}\right)+(1-a(k)) \text { Gaussian }\left(y(k), \sqrt{\sigma_{2}^{2}(k)+\sigma_{n}^{2}(k)}\right)} .
\end{aligned}
$$

Now, thresholding function (11) can be written as

$$
\begin{aligned}
\widehat{w}(k)= & \frac{a(k) \text { Gaussian }\left(y(k), \sqrt{\sigma_{1}^{2}(k)+\sigma_{n}^{2}(k)}\right)\left(\sigma_{1}^{2}(k) /\left(\sigma_{1}^{2}(k)+\sigma_{n}^{2}\right)\right) y(k)}{a(k) \text { Gaussian }\left(y(k), \sqrt{\sigma_{1}^{2}(k)+\sigma_{n}^{2}(k)}\right)+(1-a(k)) \text { Gaussian }\left(y(k), \sqrt{\sigma_{2}^{2}(k)+\sigma_{n}^{2}(k)}\right)} \\
& +\frac{(1-a(k)) \text { Gaussian }\left(y(k), \sqrt{\sigma_{2}^{2}(k)+\sigma_{n}^{2}(k)}\right) \times\left(\sigma_{2}^{2}(k) /\left(\sigma_{2}^{2}(k)+\sigma_{n}^{2}\right)\right) y(k)}{a(k) \text { Gaussian }\left(y(k), \sqrt{\sigma_{1}^{2}(k)+\sigma_{n}^{2}(k)}\right)+(1-a(k)) \text { Gaussian }\left(y(k), \sqrt{\sigma_{2}^{2}(k)+\sigma_{n}^{2}(k)}\right)} .
\end{aligned}
$$

Equation (15) is a thresholding function which can be used to shrink DTCWT coefficients. The EM algorithm is utilized to compute $\sigma_{1}(k)$ and $\sigma_{2}(k)$ [28]. A robust median estimator is an accepted way in the finest scale subband to estimate the noise standard deviation $\sigma_{n}[19,29]$.

$$
\sigma_{n}=\frac{\operatorname{median}\left(\left|y_{f}\right|\right)}{0.6745},
$$

where $y_{f}$ denotes the noisy wavelet coefficients from finest subband in scale.

However, it is difficult to determine the three parameters, since the equation $\operatorname{var}[w(k)] \neq a^{2}(k) \sigma_{1}^{2}(k)+\left(1-a^{2}(k)\right) \sigma_{2}^{2}(k)$ exits. The EM algorithm is most frequently utilized to compute the three parameters $\sigma_{1}(k), \sigma_{2}(k)$, and $a(k)$ from data [28], but it ignores the interscale dependency of the DTCWT coefficient. Considering the importance of the three parameters and the interscale dependency between subbands, it is necessary to develop an algorithm to compute parameters in the following sections.

\section{Quantum-Inspired Standard Deviation (QSD)}

Thresholding function (15) using $\sigma_{1}(k), \sigma_{2}(k)$ can reduce noise notably; however the progress of the EM algorithm ignores the interscale dependency between DTCWT subbands and $\sigma_{1}(k), \sigma_{2}(k)$ computed are CSDs in essence. Experiments show that $\sigma_{1}(k), \sigma_{2}(k)$ are not only quite different 
from scale to scale, but also different for each pixel in the same subband. The works of many authors have shown strong evidence that wavelet coefficients produced by true signal exhibit significant dependencies on subsequent locations. However, the coefficients caused by noise will decay rapidly along scales [16]. The magnitudes of noise coefficients have weaker correlation across the wavelet decomposition scales than those of true signal coefficients. Estimating a true signal by analyzing interscale coefficients has been widely used in denoising algorithms. Therefore, in this section, the interscale dependency of wavelet coefficient across scales is combined to further process $\sigma_{1}(k), \sigma_{2}(k)$ after EM algorithm to improve the denoising effects.

3.1. The Concept of Quantum Bit. The quantum bit (qubit) is the smallest unit of information in principle of quantum theory. It is a probabilistic representation which can represent a linear superposition of two ground states. The qubit is defined by a superposition of ground states $|0\rangle$ and $|1\rangle$ with a pair of numbers $(\alpha, \beta)$ as follows [30]:

$$
|\phi\rangle=\alpha|0\rangle+\beta|1\rangle,
$$

where $|\phi\rangle,|0\rangle,|1\rangle$ are the quantum states; $\alpha$ and $\beta$ denote the probability amplitudes of the corresponding states. In the representation, $|\alpha|^{2}$ and $|\beta|^{2}$ give the probability that the qubit will be in the " 0 " state and " 1 " state, respectively. So, the state of the qubit $|\phi\rangle$ varies as $\alpha$ and $\beta$ change. Therefore, a qubit can represent all states that a datum may have. A qubit may be in the "1" state, or in the " 0 " state, or in superposition of the two corresponding states with weighting factors $\alpha$ and $\beta$. Since DTCWT coefficients of a sampling signal are the superposition of noise-free coefficients and noise coefficients, it is potential to handle the information in wavelet coefficients by qubit.

$\alpha$ and $\beta$ are complex numbers, and the following normalization condition is always satisfied:

$$
|\alpha|^{2}+|\beta|^{2}=1
$$

In physics the spin-up and spin-down orientations can be the interpretations of ground states 0 and 1, and the ground state and the excited state of an atom can also represent $|0\rangle$ and $|1\rangle$, respectively [31]. As similarly known, a noise DTCWT coefficient can represent $|0\rangle$ and a true signal DTCWT coefficient can represent $|1\rangle$ when the quantum mechanics is employed to analyze the wavelet coefficients of a sampled mechanical vibration signal.

\subsection{Quantum-Inspired Standard Deviation (QSD). The} parameters $\sigma_{1}(k), \sigma_{2}(k)$ are variates in thresholding function equation (15). They not only control the shape of the function, but also influence the denoising performance. Following Section 3.1, we focus on incorporating quantum theory into the standard deviations $\sigma_{1}(k), \sigma_{2}(k)$, which are calculated by EM algorithm, to improve the thresholding effects. Then the CSDs $\sigma_{1}(k), \sigma_{2}(k)$ are converted to QSDs which are tuned by the quantum-inspired appearing probability of true signal coefficients. One QSD characterizes interscale dependency between a coefficient and its parent and achieves a degree of noise reduction for the sampled mechanical signal via thresholding function.

Noise and true signal have quite different singularity in wavelet domain. After transformation, wavelet coefficients of a true signal have strong scale dependency between each subband, especially at the point of signal saltation. However wavelet coefficients of noise decrease remarkably as decomposition level increases. These result in the fact that the product of a child coefficient and its parent coefficient for true signal is larger than the product for noise. Therefore, the product of coefficients between adjacent subbands can be used for denoising.

Considering the amount of parent coefficients is twice the amount of child coefficients, the parent-child wavelet modulus is formulated as

$$
C_{s}(k)=\left|y_{s+1}\left(\operatorname{round}\left(\frac{k}{2}\right)\right)\right| \times\left|y_{s}(k)\right|,
$$

where $s$ denotes the parent level, $s+1$ denotes the child level, and round denotes the rounding-off method. $C_{s}(k)$ denote the product of a parent wavelet modulus $\left|y_{s+1}(\operatorname{round}(k / 2))\right|$ and a child wavelet modulus $\left|y_{s}(k)\right| . C_{s}(k)$ is at the $k$ th position in the $s$ th decomposition level. Note that it is stated in Section 2.1 that $y$ in (19) refers to real part of DTCWT coefficients since we just discuss real parts. However, (19) is also applicative to imaginary part when we handle the coefficients under the imaginary domain while $y$ denotes the imaginary part.

Coefficients of a sampled signal are the composition of noise coefficients and true signal coefficients in nature, having similarity with the superposition in quantum theory. Inspired by basic quantum mechanics, the parent-child wavelet modulus $C_{s}(k)$ can be also expressed as the superposition of a noise wavelet coefficient and a true signal wavelet coefficient:

$$
\left|C_{s}(k)\right\rangle=\alpha(k)|0\rangle+\beta(k)|1\rangle,
$$

where $\alpha(k)$ and $\beta(k)$ denote the probability amplitudes of noise wavelet coefficient $|0\rangle$ and true signal wavelet coefficient, respectively. Normalize $C_{s}(k)$ as $N C_{s}(k) \in[0,1]$, and let $\alpha(k)=\cos \left(N C_{s}(k) \times \pi / 2\right), \beta(k)=\sin \left(N C_{s}(k) \times \pi / 2\right)$. The normalizing condition $\alpha^{2}(k)+\beta^{2}(k)=1$ is then satisfied, and we can write the parent-child real wavelet modulus in a quantum-inspired form

$$
\begin{aligned}
\left|C_{s}(k)\right\rangle= & \cos \left(N C_{s}(k) \times \frac{\pi}{2}\right)|0\rangle \\
& +\sin \left(N C_{s}(k) \times \frac{\pi}{2}\right)|1\rangle .
\end{aligned}
$$

In physical significance, $N C_{s}(k)$ reflects the appearing probability of true signal and noise. If the value of $N C_{s}(k)$ is less, the corresponding position between parent and child has less dependency or less energy, which means this position has larger probability for noise to appear. Conversely, if the value of $N C_{s}(k)$ is larger, the corresponding position between parent and child has lager dependency and larger energy, which means this position has larger probability for true signal to appear. 
After transforming, $\cos ^{2}\left(N C_{s}(k) \times \pi / 2\right)$ in (21) is used to represent the appearing probability of noise at the $k$ th position in the sth decomposition level and $\sin ^{2}\left(N C_{s}(k) \times\right.$ $\pi / 2)$ in (21) is used to represent the appearing probability of true signal at $k$ th position in the $s$ th decomposition level. If $N C_{s}(k)=0$, it means that the corresponding position is totally noise. If $N C_{s}(k)=1$, it means that the corresponding position is totally true signal. Combining appearing probability of true signal and noise, the estimation for $k$ th position at the present $s$ th subband is QSD

$$
\begin{aligned}
& \sigma_{1, \mathrm{QSD}}(k)=\sigma_{1} \sin \left(N C_{s}(k) \times \frac{\pi}{2}\right) \\
& \sigma_{2, \mathrm{QSD}}(k)=\sigma_{2} \sin \left(N C_{s}(k) \times \frac{\pi}{2}\right) .
\end{aligned}
$$

The proposed QSDs utilize interscale dependency between wavelet coefficients. Substituting for CSDs in the thresholding function based on SGMM, thresholding function (15) can be written as

$$
\begin{aligned}
\widehat{w}(k)= & \frac{a(k) \text { Gaussian }\left(y(k), \sqrt{\sigma_{1, \mathrm{QSD}}^{2}(k)+\sigma_{n}^{2}(k)}\right)\left(\sigma_{1, \mathrm{QSD}}^{2}(k) /\left(\sigma_{1, \mathrm{QSD}}^{2}(k)+\sigma_{n}^{2}\right)\right) y(k)}{a(k) \text { Gaussian }\left(y(k), \sqrt{\sigma_{1, \mathrm{QSD}}^{2}(k)+\sigma_{n}^{2}(k)}\right)+(1-a(k)) \text { Gaussian }\left(y(k), \sqrt{\sigma_{2, \mathrm{QSD}}^{2}(k)+\sigma_{n}^{2}(k)}\right)} \\
& +\frac{(1-a(k)) \text { Gaussian }\left(y(k), \sqrt{\sigma_{2, \mathrm{QSD}}^{2}(k)+\sigma_{n}^{2}(k)}\right) \times\left(\sigma_{2, \mathrm{QSD}}^{2}(k) /\left(\sigma_{2, \mathrm{QSD}}^{2}(k)+\sigma_{n}^{2}\right)\right) y(k)}{a(k) \text { Gaussian }\left(y(k), \sqrt{\sigma_{1, \mathrm{QSD}}^{2}(k)+\sigma_{n}^{2}(k)}\right)+(1-a(k)) \text { Gaussian }\left(y(k), \sqrt{\sigma_{2, \mathrm{QSD}}^{2}(k)+\sigma_{n}^{2}(k)}\right)} .
\end{aligned}
$$

If the QSD indicates that the appearing probability of true signal is larger, the value of the thresholding function is larger adaptively. On the contrary, if QSD indicates that the appearing probability of noise is larger, the value of the thresholding function is less adaptively. These characteristics are in favor of noise reduction. Obviously the most important point is that the quantum-inspired appearing probability of noise-free signal which is denoted as $\sin \left(N C_{s} \times \pi / 2\right)$ adaptively adjusts CSDs $\sigma_{1}(k), \sigma_{2}(k)$. The true signal of the machinery is enhanced and the noise is weakened with the thresholding function. In this thresholding function with QSD, the unimportant coefficients are shrunk nonlinearly instead of setting to zero, which can not only reduce noise but also preserve more shape details, such as impulsive feature of fault.

3.3. Procedure of Denoising Algorithm with QSD Based on $S G M M$. The denoising of the mechanical vibration signals employing QSD in thresholding function based on SGMM can be done in the following way:

(1) Sample a mechanical vibration signal.

(2) Decompose the noisy mechanical vibration signal by DTCWT.

(3) Threshold the real part of the DTCWT coefficients using (23) in each subband.

(4) Threshold the imaginary part of the DTCWT coefficients using (23) in each subband.

(5) Apply the inverse DTCWT to the thresholded coefficients to obtain the denoised signal. The flow chart of the algorithm is shown in Figure 1.

\section{Application for Bearing Signal Denoising}

4.1. Experimental System of Rolling Element Bearing. The mechanical vibration signal used in this paper was acquired from a mechanical power-shift steering transmission. The test stand consists of a reliance electric motor, an accelerometer located on tank cover over the fault bearing with a magnetic base, and a data recorder with a sampling frequency of $10000 \mathrm{~Hz}$ per channel. The single point fault was set for the rolling element bearing at the inner raceway using electrodischarge machining with fault diameter of $3 \mathrm{~mm}$ and fault depth of $2 \mathrm{~mm}$. The shaft rotational speed was $1830 \mathrm{rpm}$ $(30.5 \mathrm{~Hz})$ while the equipment was at third gear. The time of sampling was 0.15 seconds [32]. On the basis of the theoretical calculation the characteristic frequency of inner race fault is $158 \mathrm{~Hz}$. The waveform of the analyzed signal in the time domain and the frequency spectrum are displayed in Figure 2. The defect frequency $158 \mathrm{~Hz}$ cannot be detected in Figure 2(b). Both the waveform and the spectrum indicate that the signal is corrupted by strong noise.

4.2. Applying for Denoising. The presented denoising method using QSD based on SGMM is employed to reduce noise and the results are shown in Figure 3. Since the soft thresholding technique and hard thresholding technique are among the most widely used thresholding methods to depress noise, they are employed for purposes of comparison with the proposed algorithm. The results of soft and hard thresholding technique are shown in Figures 4 and 5.

(1) In the Time Domain. The soft and hard thresholding technique remove most of the noise, but they also remove the useful signal, which leads to the fact that the shock period is incorrect. The denoised signal by denoising method using QSD based on SGMM has more impact information, which is important for fault diagnosis. The proposed denoising method can depress noise and maintain the impulsive components. 


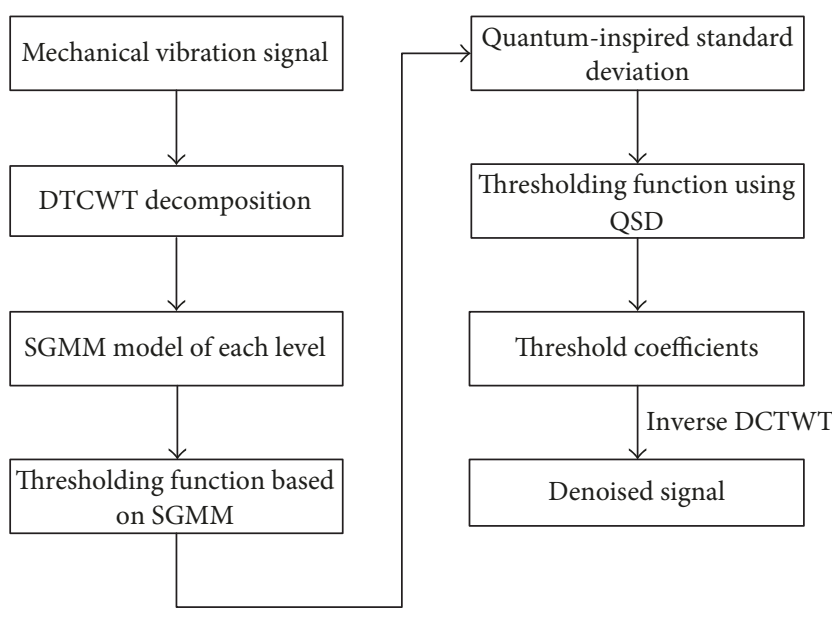

FIGURE 1: Flow chart of the proposed denoising algorithm.

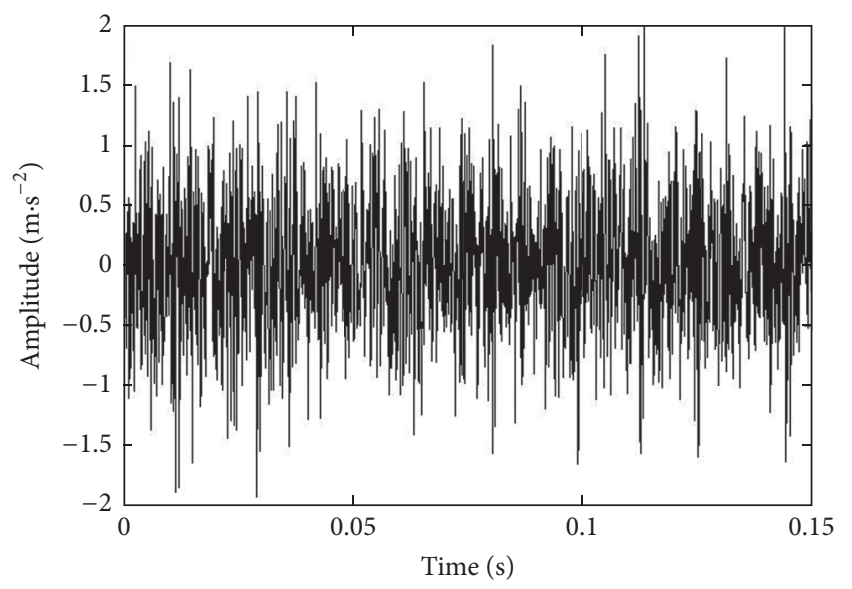

(a)

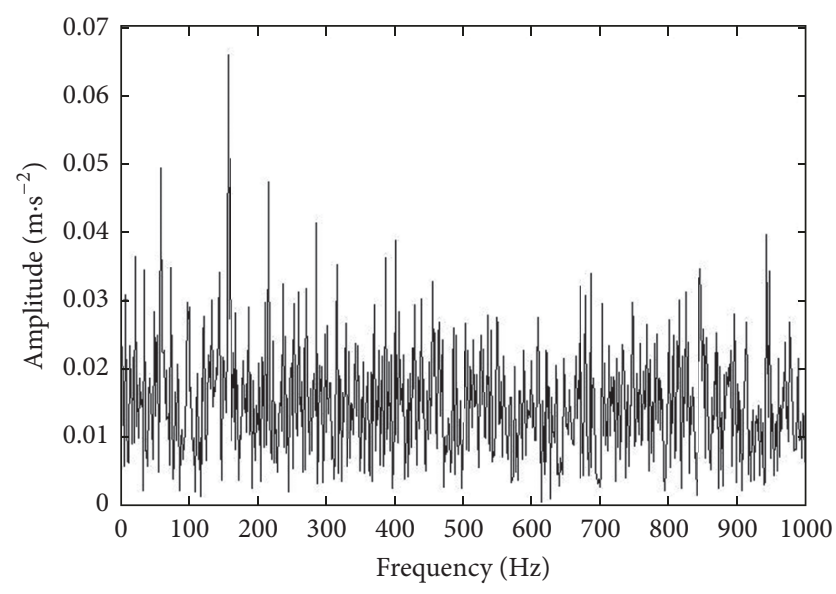

(b)

FIGURE 2: Fault signal with strong noise: (a) waveform in the time domain; (b) frequency spectrum.

(2) In the Frequency Domain. The characteristic frequency $158 \mathrm{~Hz}$ can be seen in Figures 3(b), 4(b), and 5(b), which reveals that the three thresholding methods are useful. There are two obvious features of rolling element bearing with inner race fault: (1) The characteristic frequency and its harmonic frequencies have sideband and the difference between the sideband and its center frequency is equal to the rotating frequency. (2) The rotating frequency and its harmonic frequencies are significant. However the characteristic frequency has no correct sideband and the spectra have no harmonic frequencies after soft and hard thresholding. The characteristic frequency $158 \mathrm{~Hz}$ and its harmonic frequency at $316 \mathrm{~Hz}$ can be identified clearly in Figure 3(b) and side frequencies $(158 \pm 30.5 \mathrm{~Hz}, 316 \pm 30.5 \mathrm{~Hz})$ are prominent. Furthermore, the difference between the side frequencies and the center frequency is $30.5 \mathrm{~Hz}$ which is equal to the rotating frequency. In addition, in Figure 3(b) the modulation frequencies $30.5 \mathrm{~Hz}$ (equal to the frequency of rotor rotating) and $61 \mathrm{~Hz}$ (equal to the second harmonic frequency of rotor rotating) are very clear too. For all of these characteristics accord well with the characteristics of a standard bearing spectrum with inner race fault, we can confirm that there is an inner race fault in the rolling element bearing.

\section{Conclusions}

There are two highlights of the proposed denoising technique in this paper: (1) The SGMM is proposed and utilized to approximate the local PDF of each wavelet coefficient. The thresholding function is derived after the MAP estimation using the SGMM as the prior knowledge, which extends the application of the GMM to one-dimensional mechanical signal. (2) The quantum theory is employed to describe the interscale dependency of wavelet coefficients and the QSD is presented. Experimental results show that the denoising technique using the SGMM and QSD can achieve better denoising performance in both the time and frequency domains. The success of the denoising algorithm depends upon the ability of the SGMM modeling the statistical distribution accurately and the QSD exploring the interscale dependency of adjacent subbands correctly and efficiently. The algorithm is tested and verified through a mechanical vibration signal with bearing fault discussed as a denoising case in Section 4. The advantages of the proposed denoising algorithm becomes more noticeable when the mechanical vibration signal is corrupted by more noise.

Furthermore, instead of this thresholding function derived from the SGMM by the MAP estimator, the QSD can be applied into other nonlinear thresholding functions, for example, generalized Gaussian distribution based thresholding function or the MMSE estimation based thresholding function. However, these methods take longer time which may be more applicable for some signal processing.

\section{Conflicts of Interest}

The authors declare that they have no conflicts of interest. 


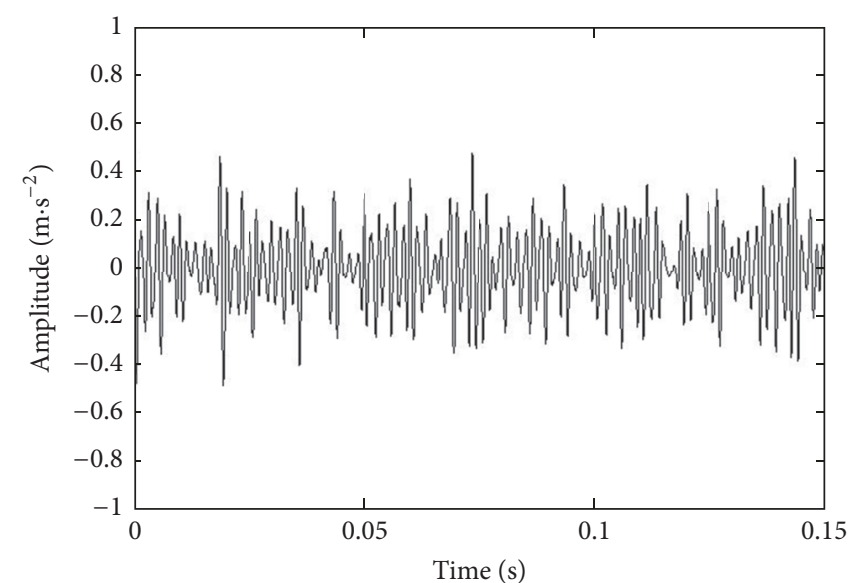

(a)

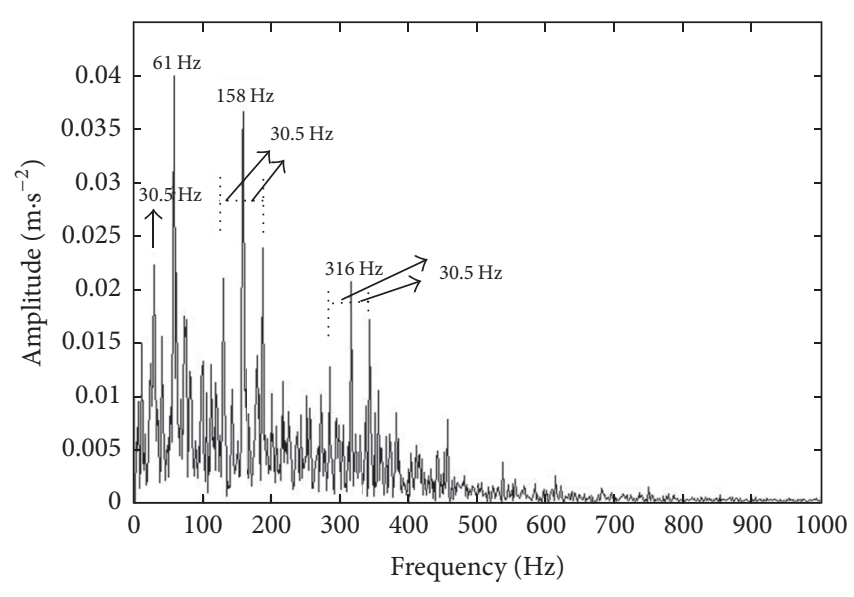

(b)

FIGURE 3: Denoising by thresholding function using QSD based on SGMM: (a) waveform in the time domain; (b) frequency spectrum.

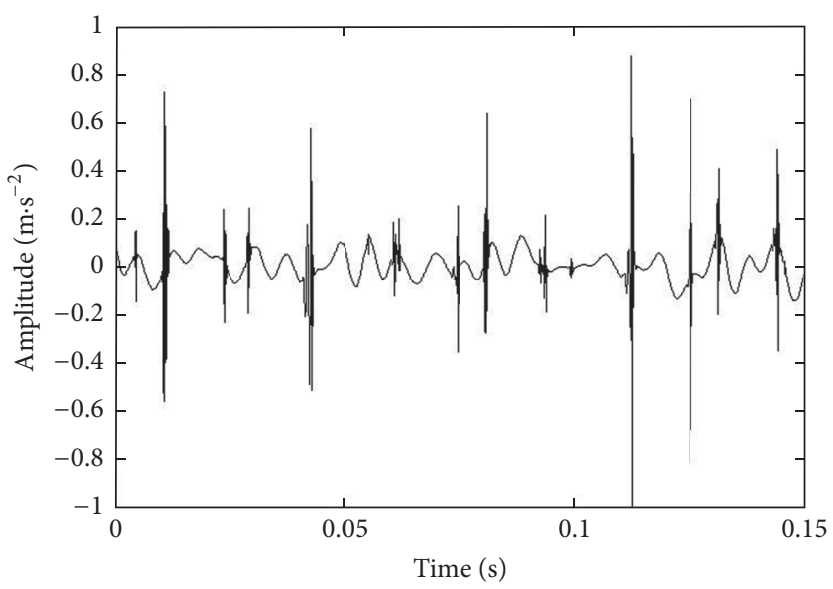

(a)

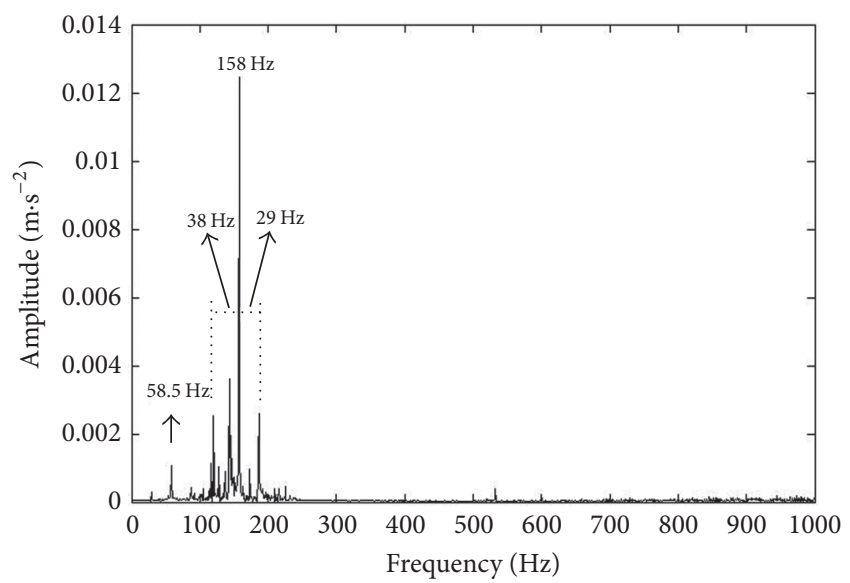

(b)

FIGURE 4: Denoising by soft thresholding technique: (a) waveform in the time domain; (b) frequency spectrum.

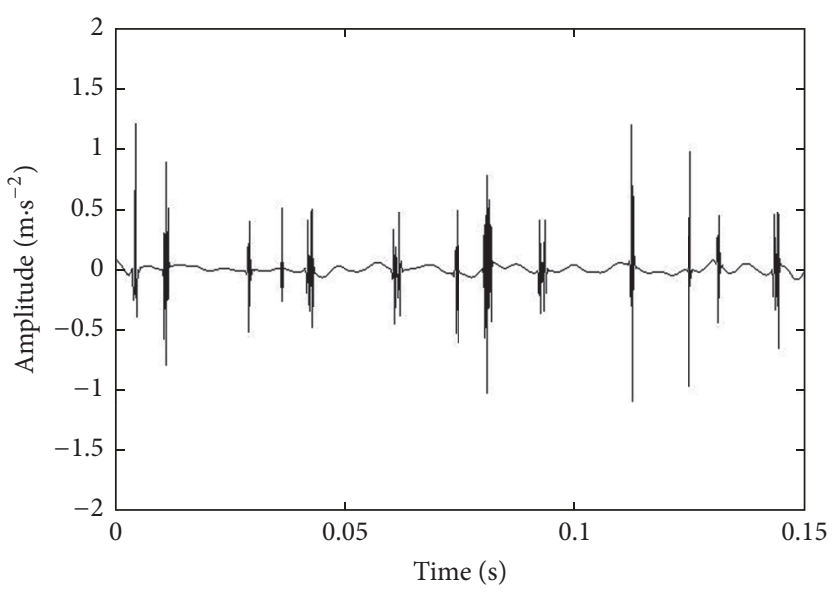

(a)

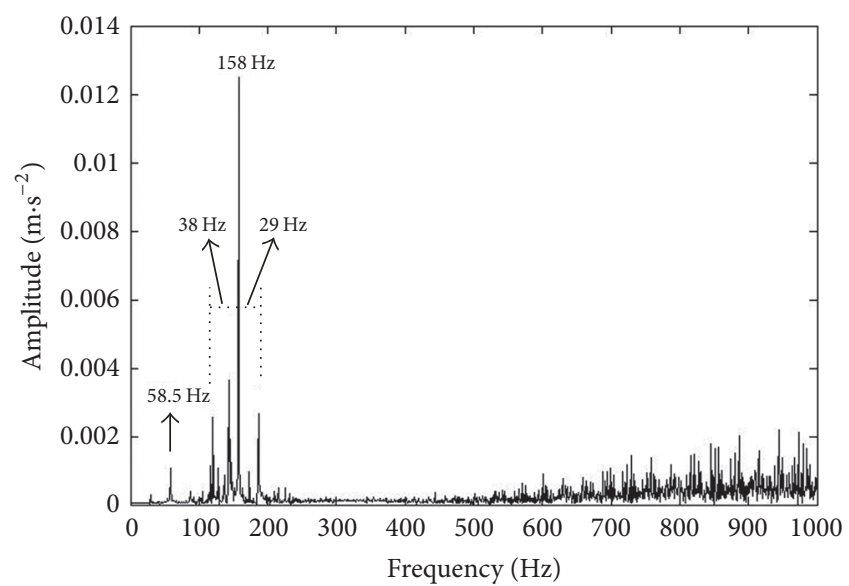

(b)

FIGURE 5: Denoising by hard thresholding technique: (a) waveform in the time domain; (b) frequency spectrum. 


\section{References}

[1] H. Zhang, X. Zhang, and J. Wang, "Robust gain-scheduling energy-to-peak control of vehicle lateral dynamics stabilisation," Vehicle System Dynamics, vol. 52, no. 3, pp. 309-340, 2014.

[2] H. Zhang, J. Wang, and Y. Shi, "Robust $\mathrm{H}_{\infty}$ sliding-mode control for Markovian jump systems subject to intermittent observations and partially known transition probabilities," Systems \& Control Letters, vol. 62, no. 12, pp. 1114-1124, 2013.

[3] Y. Shi, H. Zhang, and J. Wang, "On energy-to-peak filtering for nonuniformly sampled nonlinear systems: a Markovian jump system approach," IEEE Transactions on Fuzzy Systems, vol. 22, no. 1, pp. 212-222, 2014.

[4] H. Zhang, Y. Shi, and B. Mu, "Optimal $\mathrm{H}_{\infty}$-based linearquadratic regulator tracking control for discrete-time takagisugeno fuzzy systems with preview actions," Journal of Dynamic Systems, Measurement, and Control, vol. 135, no. 4, Article ID 044501, 2013.

[5] Z. Shuai, H. Zhang, J. Wang, J. Li, and M. Ouyang, "Lateral motion control for four-wheel-independent-drive electric vehicles using optimal torque allocation and dynamic message priority scheduling," Control Engineering Practice, vol. 24, no. 1, pp. 55-66, 2014.

[6] M. A. Kabir and C. Shahnaz, "Denoising of ECG signals based on noise reduction algorithms in EMD and wavelet domains," Biomedical Signal Processing and Control, vol. 7, no. 5, pp. 481489, 2012.

[7] Y. Wang, Z. He, and Y. Zi, "Enhancement of signal denoising and multiple fault signatures detecting in rotating machinery using dual-tree complex wavelet transform," Mechanical Systems and Signal Processing, vol. 24, no. 1, pp. 119-137, 2010.

[8] C.-C. Liu, T.-Y. Sun, S.-J. Tsai, Y.-H. Yu, and S.-T. Hsieh, "Heuristic wavelet shrinkage for denoising," Applied Soft Computing, vol. 11, no. 1, pp. 256-264, 2011.

[9] J. Saeedi, M. H. Moradi, and K. Faez, "A new wavelet-based fuzzy single and multi-channel image denoising," Image and Vision Computing, vol. 28, no. 12, pp. 1611-1623, 2010.

[10] M. Nasri and H. Nezamabadi-pour, "Image denoising in the wavelet domain using a new adaptive thresholding function," Neurocomputing, vol. 72, no. 4-6, pp. 1012-1025, 2009.

[11] G. Andria, F. Attivissimo, G. Cavone, N. Giaquinto, and A. M. L. Lanzolla, "Linear filtering of 2-D wavelet coefficients for denoising ultrasound medical images," Measurement, vol. 45, no. 7, pp. 1792-1800, 2012.

[12] V. Soni, A. K. Bhandari, A. Kumar, and G. K. Singh, "Improved sub-band adaptive thresholding function for denoising of satellite image based on evolutionary algorithms," IET Signal Processing, vol. 7, no. 8, pp. 720-730, 2013.

[13] S. Khullar, A. Michael, N. Correa, T. Adali, S. A. Baum, and V. D. Calhoun, "Wavelet-based fMRI analysis: 3-D denoising, signal separation, and validation metrics," NeuroImage, vol. 54, no. 4, pp. 2867-2884, 2011.

[14] N. R. Ghugre, M. Martin, M. Scadeng et al., "Superiority of 3D wavelet-packet denoising in MR microscopy," Magnetic Resonance Imaging, vol. 21, no. 8, pp. 913-921, 2003.

[15] H. Rabbani and M. Vafadust, "Image/video denoising based on a mixture of Laplace distributions with local parameters in multidimensional complex wavelet domain," Signal Processing, vol. 88, no. 1, pp. 158-173, 2008.

[16] U. D. Dwivedi and S. N. Singh, "Enhanced detection of power-quality events using intra and interscale dependencies of wavelet coefficients," IEEE Transactions on Power Delivery, vol. 25, no. 1, pp. 358-366, 2010.

[17] J. Yang, W. Xu, and Q. Dai, "Fast adaptive wavelet packets using interscale embedding of decomposition structures," Pattern Recognition Letters, vol. 31, no. 11, pp. 1481-1486, 2010.

[18] S. Jazebi, B. Vahidi, S. H. Hosseinian, and J. Faiz, "Magnetizing inrush current identification using wavelet based gaussian mixture models," Simulation Modelling Practice and Theory, vol. 17, no. 6, pp. 991-1010, 2009.

[19] S. Kayhan and E. Erçelebi, "ECG denoising on bivariate shrinkage function exploiting interscale dependency of wavelet coefficients," Turkish Journal of Electrical Engineering \& Computer Sciences, vol. 19, no. 3, pp. 495-511, 2011.

[20] T.-C. Lu and G.-R. Yu, "An adaptive population multi-objective quantum-inspired evolutionary algorithm for multi-objective 0/1 knapsack problems," Information Sciences, vol. 243, pp. 3956, 2013.

[21] A. Layeb, "A hybrid quantum inspired harmony search algorithm for 0-1 optimization problems," Journal of Computational and Applied Mathematics, vol. 253, pp. 14-25, 2013.

[22] P. Li, "A quantum-behaved evolutionary algorithm based on the Bloch spherical search," Communications in Nonlinear Science and Numerical Simulation, vol. 19, no. 4, pp. 763-771, 2014.

[23] Y. Li, H. Shi, L. Jiao, and R. Liu, "Quantum evolutionary clustering algorithm based on watershed applied to SAR image segmentation," Neurocomputing, vol. 87, pp. 90-98, 2012.

[24] J. S. Shaari and A. A. Bahari, "Improved two-way six-state protocol for quantum key distribution," Physics Letters A, vol. 376, no. 45, pp. 2962-2966, 2012.

[25] J. D. Nelson and N. G. Kingsbury, "Fractal dimension, wavelet shrinkage and anomaly detection for mine hunting," IET Signal Processing, vol. 6, no. 5, pp. 484-493, 2012.

[26] G. Chen and W.-P. Zhu, "Signal denoising using neighbouring dual-tree complex wavelet coefficients," IET Signal Processing, vol. 6, no. 2, pp. 143-147, 2012.

[27] Z. Yao and W. Liu, "Extracting robust distribution using adaptive Gaussian Mixture Model and online feature selection," Neurocomputing, vol. 101, pp. 258-274, 2013.

[28] L. Chen, A. Tulsyan, B. Huang, and F. Liu, "Multiple model approach to nonlinear system identification with an uncertain scheduling variable using em algorithm," Journal of Process Control, vol. 23, no. 10, pp. 1480-1496, 2013.

[29] D. L. Donoho, "De-noising by soft-thresholding," IEEE Transactions on Information Theory, vol. 41, no. 3, pp. 613-627, 1995.

[30] P. C. Li, K. P. Song, and F. H. Shang, "Double chains quantum genetic algorithm with application to neuro-fuzzy controller design," Advances in Engineering Software, vol. 42, no. 10, pp. 875-886, 2011.

[31] J. Tsai, F.-Y. Hsiao, Y.-J. Li, and J.-F. Shen, "A quantum search algorithm for future spacecraft attitude determination," Acta Astronautica, vol. 68, no. 7-8, pp. 1208-1218, 2011.

[32] Y. Chen, P. Zhang, Z. Wang, W. Yang, and Y. Yang, "Denoising algorithm for mechanical vibration signal using quantum Hadamard transformation," Measurement, vol. 66, pp. 168-175, 2015. 


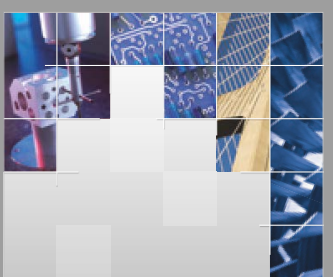

\section{Enfincering}
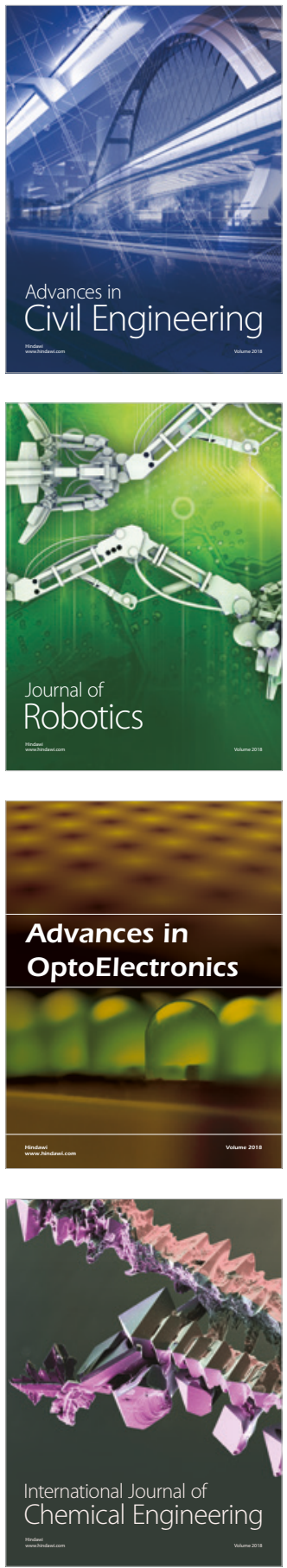

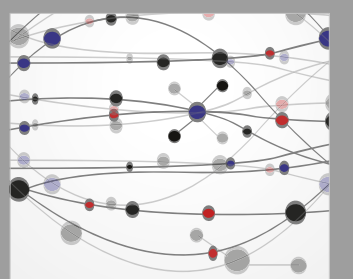

\section{Rotating \\ Machinery}

The Scientific World Journal

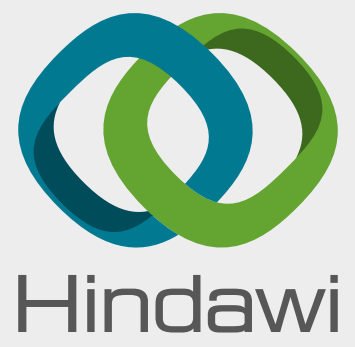

Submit your manuscripts at

www.hindawi.com
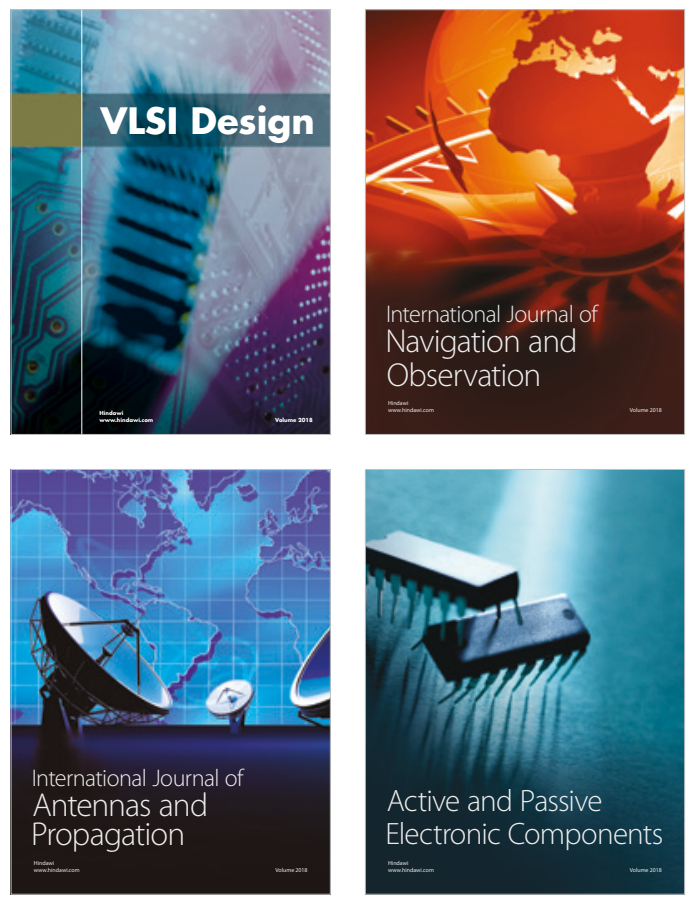
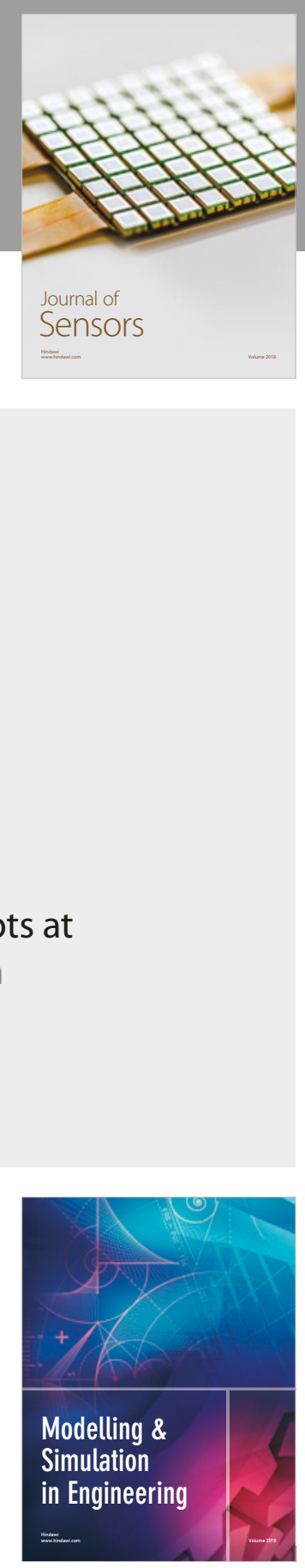

\section{Advances \\ Multimedia}
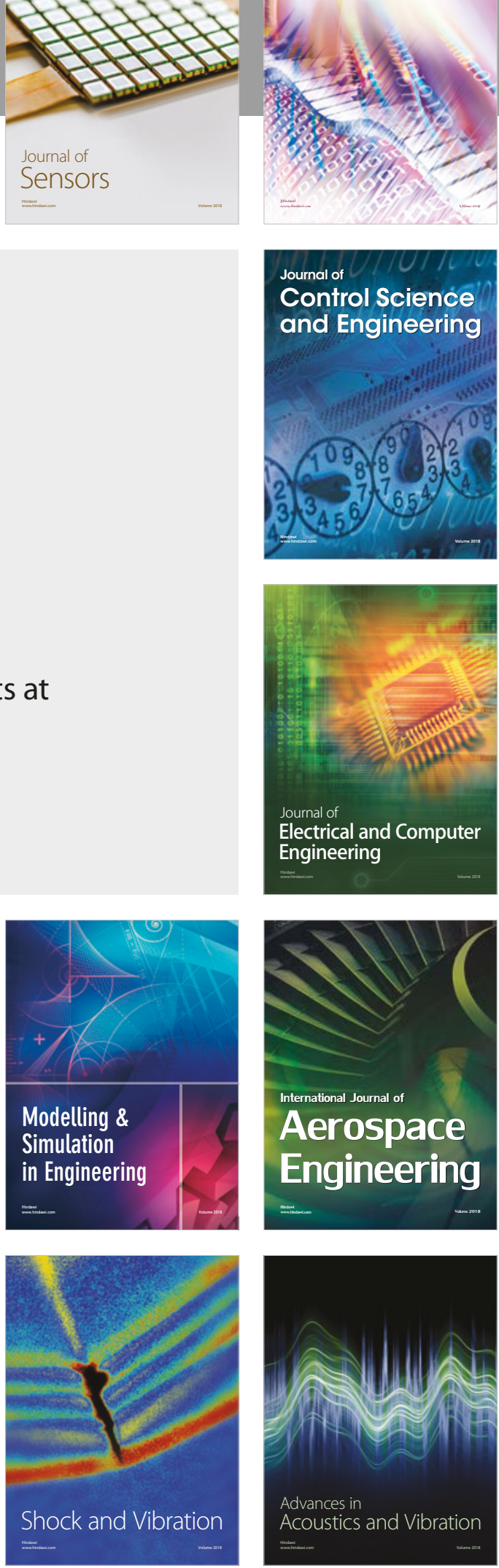